\title{
Uncertainty Due to Unsteady Fluid/Structure Interaction for the Ares I Vehicle Traversing the Transonic Regime
}

\author{
Robert E. Bartels* \\ NASA Langley Research Center, Hampton, VA, 23681, USA
}

\begin{abstract}
Rapid reduced-order numerical models are being investigated as candidates to simulate the dynamics of a flexible launch vehicle during atmospheric ascent. There has also been the extension of these new approaches to include gust response. These methods are used to perform aeroelastic and gust response analyses at isolated Mach numbers. Such models require a method to time march through a succession of ascent Mach numbers. An approach is presented for interpolating reduced-order models of the unsteady aerodynamics at successive Mach numbers. The transonic Mach number range is considered here since launch vehicles can suffer the highest dynamic loads through this range. Realistic simulations of the flexible vehicle behavior as it traverses this Mach number range are presented. The response of the vehicle due to gusts is computed. Uncertainties in root mean square and maximun bending moment and crew module accelerations are presented due to assumed probability distributions in design parameters, ascent flight conditions, gusts. The primary focus is on the uncertainty introduced by modeling fidelity. It is found that an unsteady reduced order model produces larger excursions in the root mean square loading and accelerations than does a quasi-steady reduced order model.
\end{abstract}

\section{Nomenclature}

$A$
$b_{g}$
$g$
$g_{s}$
$G_{g}$
$G_{e d}$
$G_{e s}$
$\kappa$
$K$
$L_{g_{n}}$
$M$
$N_{R}$
$q_{\infty}$
$q_{\text {nom }}$
$Q_{k}$
$Q_{m}$
$Q_{\zeta}$
$S_{n}$
$T_{\text {zed }}$
$T_{\text {zes }}$
$t_{g_{n}}$
$U_{\infty}$
$U_{\text {nom }}$
$w_{g}$
$w_{\text {ref }}$

$=$ State matrix

$=$ Gust model scale factor

$=$ Generalized displacement

$=$ Static or mean generalized displacement

$=$ Generalized force due to gust

$=$ Generalized force due to dynamic component of gimballed engine thrust

$=$ Generalized force due to mean component of gimballed engine thrust

$=$ Fractional exponent

$=$ Structural stiffness matrix

$=$ Gust ramp width of component $n$, grid units

$=$ Structural and aerodynamic mass matrix

$=$ Number of lag states, $N_{R}=10$

$=$ Free stream dynamic pressure, $p s i$

$=$ Free stream dynamic pressure for nominal trajectory, $p s i$

$=$ Generalized aerodynamic stiffness matrix

$=$ Generalized aerodynamic mass matrix

$=$ Generalized aerodynamic damping matrix

$=$ Unit sign function $( \pm 1)$

$=$ Dynamic $z$-component of engine thrust, $l b s$

$=$ Mean $z$-component of engine thrust, $l b s$

$=$ Gust time start of component $n, \mathrm{sec}$

$=$ Free stream velocity, $\mathrm{in} / \mathrm{sec}$

$=$ Free stream velocity for nominal trajectory, in $/ \mathrm{sec}$

$=$ Nondimensional vertical gust velocity

$=$ Vertical gust reference magnitude of component $n, \mathrm{in} / \mathrm{sec}$

${ }^{*}$ Senior Research Engineer, Aeroelasticity Branch, AIAA Senior Member

$$
1 \text { of } 16
$$




$\begin{array}{ll}x_{\tau}, y_{\tau}, z_{\tau} & =\text { Nondimensional grid speed metrics } \\ x_{g} & =x \text { location of the engine gimbal, in } \\ \tilde{x}_{\tau}, \tilde{y}_{\tau}, \tilde{z}_{\tau} & =\text { Modified nondimensional grid speed metrics } \\ \alpha & =\text { Angle of attack, degrees } \\ \alpha_{l} & =\text { Local angle of attack, degrees } \\ \beta_{l} & =\text { Local angle of sideslip, degrees } \\ \gamma & =\text { Roger approximation lag root matrix } \\ \Delta & =\text { Structural and aerodynamic damping matrix } \\ \Delta \alpha_{g} & =\text { Local angle of attack increment due to gust, radians } \\ \phi & =\text { Matrix of eigenvectors of structural dynamics equations } \\ \Phi_{n}(x) & =\text { Functional representation of structural eigenvector } n \text { projected to vehicle centerline } \\ \chi & =\text { State variable matrix } \\ \xi & =\text { Unsteady aerodynamic lag state } \\ \zeta_{s} & =\text { Structural damping ratio } \\ \zeta_{a} & =\text { Aerodynamic damping ratio } \\ \tau & =\text { Nondimensional CFD time } \\ \Omega & =\text { Structural and aerodynamic stiffness matrix }\end{array}$

Note to Readers: The predicted performance and certain other features and characteristics of the Ares I and Ares I-X launch vehicles are defined by the U.S. Government to be Sensitive but Unclassified (SBU). Therefore, details have been removed from all plots and figures and tabulated data may have been rescaled.

\section{Introduction}

Recent interest has been directed toward developing rapid reduced-order numerical models (ROM's) to simulate the behavior of flexible launch vehicles during atmospheric ascent. For example Capri, Mastroddi and Pizzicaroli use system identification of inviscid unsteady aerodynamics to perform aeroelastic stability analysis of the VEGA European small launch vehicle ${ }^{1}$. Silva performs system identification to extract a state representation of the unsteady aerodynamics of the NASA Ares I Crew Launch Vehicle (CLV) and Ares I-X Flight Test Vehicle (FTV) ${ }^{2}$. These reduced-order models linearize the unsteady aerodynamics about a mean nonlinear Computational Fluid Dynamics (CFD) generated flow field. There has also been the extension of this approach to include gust response, as for instance in the reduced-order gust model and gust response of a launch vehicle performed by Mastroddi et al. ${ }^{3}$. However, these methods are used to perform aeroelastic and gust response analyses at isolated Mach numbers. Launch vehicles intended for orbital insertion never remain at a given atmospheric flight condition for a significant time. What will also be necessary is the capability to simulate flexible vehicle dynamics through a continuous succession of ascent conditions.

While the use of aircraft and launch vehicle databases at multiple flight conditions is not new (e.g. Chen et al. ${ }^{4}$ ), it seems that the use of steady and unsteady aerodynamic databases to simulate launch vehicle ascent is new. The present paper offers one method for interpolating reduced-order models of the unsteady aerodynamics at successive Mach numbers. In this case, rational function approximations (RFA's) at multiple flight conditions are used in conjunction with nonlinear steady databases. The interest in simulating through successive conditions is not unique. Indeed, there are current efforts toward combining reduced-order models at successive flight conditions, at least in the case of aircraft ${ }^{5-7}$. In the present case the requirements may be somewhat simplified by limited consideration only of launch vehicles. Furthermore, enhancement of methods in current industry use has certain advantages. Therefore, an approach is shown in which the RFA's at key Mach numbers can be interpolated to the current flight condition. Note that the present simulations do not include the effect of rigid body degrees of freedom. In this paper the aeroelastic responses of the vehicle to gust excitation are presented. Since the engine input required for vehicle attitude control is one of the largest contributors to bending moment, engine thrust vectoring is used to instantaneously trim the vehicle at all times during the ascent.

Slight variances in the manufacture inherently result in geometric and/or material uncertainties. If probabilities can be assigned, and the physics clearly understood, uncertainties in an output of interest can be estimated. It may be possible to assign probability distributions to design or manufacture-related quantities. Probabilistic distributions in structural damping can be due to varying mechanical properties of joints and material irregularities. Numerous recent

$$
2 \text { of } 16
$$


examples of the quantification of structural related uncertainties and risks can be cited ${ }^{8-14}$. Uncertainties in flow field quantities have also been recently investigated for a variety of flight regimes ${ }^{15-18}$. Probabilities derived from statistical atmospheric data can be assigned to flight conditions such as dynamic pressure, free stream speed or to atmospheric turbulence intensities and frequency.

Uncertainties in mass and material stiffnesses can impact mode frequencies and mode shapes and ultimately the aeroelastic behavior of a structure. Uncertainties in aeroelastic stability, and in particular flutter onset, have recently been calculated for the AGARD 445.6 wing ${ }^{19}$. Beyond the calculation of flutter onset, probabilistic aeroelastic data also result in static and dynamic flight load, guidance, navigation and control and in ride quality uncertainties. Uncertainties can also be associated with modeling fidelity. In traditional loads analysis of flexible launch vehicles, rigid steady line loads are frequently used; but it is known that this method can produce results that are unconservative ${ }^{20}$. To reduce this uncertainty, various methods of higher fidelity are available, the highest being computational aeroelastic analysis using a CFD code. A compromise between these modeling approaches, namely a reduced-order model, is used in the present paper that includes the effect of unsteady flow physics.

As an example of the use of the multi-flight condition reduced-order model, the uncertainties of several quantities of interest are calculated for the flexible Ares I Crew Launch Vehicle as it traverses the transonic regime. The transonic flight regime is chosen because it is frequently the speed range through which static and dynamic flight loads vary most rapidly. The aeroelastic excitation mechanism is atmospheric gusts. Here the uncertainty is considered due to a variety of design and flight factors that influence unsteady flexible vehicle response. The design factors are structural damping and frequencies. With regard to the flight conditions, the dynamic pressure, gust shapes and velocities are given assumed probability distributions. The parameter space encompassing all these will be simulated. The resulting uncertainties in the vehicle bending moment and crew module acceleration will be assessed. These uncertainties will be computed with an unsteady ROM and with a quasi-steady ROM, thus providing an additional assessment of uncertainty due to model fidelity.

This paper will begin by discussing the implementation of gust modeling in the CFD code FUN3D. The development of the multi-flight condition dynamic and quasi-steady ROM's and their verification will be discussed. Gust modeling for a launch vehicle ascent will briefly be discussed. Finally, these methods will be applied to uncertainty quantification for parameters of interest for the Ares I launch vehicle.

\section{FUN3D Code}

\section{A. Basic Solver}

The Navier-Stokes code used in this study is the Fully Unstructured Navier-Stokes Three-Dimensional FUN3D code, a finite-volume unstructured CFD code for either compressible or incompressible flows ${ }^{21,22}$. Flow variables are stored at the vertices of the grid. FUN3D can solve the discrete compressible Euler or Reynolds-averaged Navier-Stokes (RANS) flow equations either tightly or loosely coupled with a turbulence model on mixed element grids, including tetrahedra, prisms, pyramids and hexahedra. In the present study the RANS solver and the loosely coupled SpalartAllmaras turbulence model ${ }^{23}$ are used on an all tetrahedron grid. FUN3D employs an implicit upwind algorithm in which the inviscid fluxes are obtained with a flux-splitting scheme. The low dissipation flux splitting scheme for the inviscid flux construction, and the blended Van Leer flux limiter were used ${ }^{24}$. The full Navier-Stokes viscous terms are used ${ }^{21}$. Steady state solutions are accelerated to convergence by the use of local time stepping ${ }^{25}$. Domain decomposition enables distributed computing on multiple processors.

\section{B. Field Velocity Method of Modeling a Gust}

Recent innovations have allowed the simulation of a gust with a CFD code using the Field Velocity Method (FVM ${ }^{8,26-28}$. The FVM physically introduces gusts into a CFD code by utilizing grid velocity. ${ }^{28}$ Normally grid velocity would be associated with physically moving the grid. However, it is possible to introduce an arbitrary perturbation velocity in a stationary grid by prescribing the grid velocity at either all or some of the field grid points without actually moving the grid. If the gust shape is defined by a streamwise variation in a perturbation velocity, expressed as

$$
\begin{gathered}
w_{g}(x, t)=f\left(t-x / U_{\infty}\right) \quad, \quad t>x / U_{\infty} \\
3 \text { of } 16
\end{gathered}
$$


the gust profile would be introduced into the flow field by modified grid speed metrics

$$
\tilde{x}_{\tau} \hat{i}+\tilde{y}_{\tau} \hat{j}+\tilde{z}_{\tau} \hat{k}=x_{\tau} \hat{i}+y_{\tau} \hat{j}+\left(z_{\tau}-w_{g}\right) \hat{k}
$$

To date this method has been used for relatively simple to moderately complex configurations. ${ }^{29}$ Further use and development of the method have been performed by Singh and Baeder ${ }^{8}$, Zaide and Raveh ${ }^{27,30}$, Raveh ${ }^{29,31}$ and Wang et al. ${ }^{32}$.

This approach has been implemented in the FUN3D CFD code. Gust profiles such as sharp edged or compound sequences of one-minus-cosine increments are used in the present paper. A CFD calculation incorporating a gust in this fashion will be compared with a gust introduced into the dynamic reduced-order launch vehicle model.

\section{Reduced Order Models}

In order to clarify what is new, the discussion here will focus on the flight condition interpolation, gust input and response, and engine trim. The details of the method for isolated flight conditions are discussed elsewhere ${ }^{20,33,34}$. In reference [20], the solution is partitioned into linearized dynamic and nonlinear quasi-steady mean components. The dynamic solution is linearized about the nonlinear mean aeroelastic solution. The mean solution is obtained using equation 11 of reference [33] and steady state CFD generated aerodynamic line loads at Mach numbers 1.00, 1.20 and 1.55. Line loads are linearly interpolated to ascent Mach number, $\alpha_{l}$ and $\beta_{l}$ to compute the generalized forcing. The quasi-static mean equations can be written

$$
[I] g_{s}^{\prime \prime}+[2 \omega] g_{s}^{\prime}+\left[\omega^{2}\right]\left\{g_{s}\right\}=\left\{G_{s}\right\}+\left\{G_{e s}\right\}
$$

where $G_{s}$ is the generalized forcing due to aerodynamic line loads. The primes in equation 3 refer to differentiation with respect to a pseudo time used to converge the static mean problem. This paper also includes generlized force $G_{e s}$ due to the mean transverse gimballed engine forcing, where for mode $n, G_{e s n}=T_{z s} \Phi_{n}\left(x_{g}\right)$. Engine thrust is used to trim the mean component of the vehicle pitch moment.

The dynamic response of the vehicle is computed by linearizing the lineloads around the local mean $\alpha_{l}, \beta_{l}$ and static generalized force $G_{s}$. A state space model of the aeroelastic system with lag states $(\xi)$ is developed by using RFA theory. The state space model is given by

$$
\{\dot{\chi}\}=[A]\{\chi\}+\left\{G_{g}\right\}+\left\{G_{e d}\right\} \quad, \quad\{\chi\}=\{g, \dot{g}, \xi\}^{T}
$$

where

$$
[A]=\left[\begin{array}{ccc}
0 & I & 0 \\
-M^{-1} \Omega & -M^{-1} \Delta & M^{-1} q_{\infty} d \\
0 & e & \gamma
\end{array}\right]
$$

and

$$
\left[M\left(M_{\infty}\right)\right]=I-\rho_{\infty}\left[Q_{m}\left(M_{\infty}\right)\right] \quad, \quad\left[\Omega\left(M_{\infty}\right)\right]=\left[\omega^{2}\right]-\rho_{\infty} V_{\infty}^{2}\left[Q_{k}\left(M_{\infty}\right)\right] \quad, \quad\left[\Delta\left(M_{\infty}\right)\right]=\left[2 \zeta_{s} \omega\right]-\rho_{\infty} V_{\infty}\left[Q_{\zeta}\left(M_{\infty}\right)\right] .
$$

$G_{e d}$ is the component of engine thrust required to trim the gust forcing, for mode $n$ given by $G_{e d n}=T_{z d} \Phi_{n}\left(x_{g}\right) . G_{g}$ on the right hand side of equation 4 is a vector of generalized forces due to gust. Gust modeling will be discussed in the next section. Through the aerodynamic mass $\left(Q_{m}\left(M_{\infty}\right)\right)$, damping $\left(Q_{\zeta}\left(M_{\infty}\right)\right)$ and stiffness $\left(Q_{k}\left(M_{\infty}\right)\right)$ matrices and dynamic pressure $\left(q_{\infty}\right)$, the matrices $M\left(M_{\infty}\right), \Omega\left(M_{\infty}\right)$ and $\Delta\left(M_{\infty}\right)$ are functions of Mach number. The line loads also vary with Mach number. Because of propellant burn, structural frequencies $(\omega)$ and mode shapes are functions of time into ascent. As discussed in reference [20] modes 1 and 2 are modeled in a fully unsteady sense using an RFA approximation. The unsteady aerodynamic submatrix is obtained via the Roger approximation ${ }^{35}$. This approximation results in equations 12 and 13 of references [20,36]. The remaining modes are modeled with quasi-steady aerodynamics using equations 14 and 15 of reference [33]. Definitions of terms in these equations are found elsewhere ${ }^{33}$. In this paper, the structural and aerodynamic equations at Mach numbers 1.00, 1.20 and 1.55 are used to simulate the ascent of the vehicle through this Mach number range. Based on trajectory data, the steady and unsteady databases at these Mach numbers are interpolated to each time step. The constant matrix $e$ is defined in references [20,36].

To create a system that can traverse several Mach numbers in a stable manner, it was found essential to enforce the constraint that the same values of $\gamma$ are used for all Mach numbers. Therefore, 10 lag roots at Mach 1.00 were

$$
4 \text { of } 16
$$


obtained and the same used at all Mach numbers, 1.00, 1.20, and 1.55. Using the same Mach 1.00 lag roots $(\gamma)$, unsteady aerodynamic models $\left(d, Q_{k}, Q_{\zeta}\right.$, and $\left.Q_{m}\right)$ were obtained at Mach numbers 1.20 and 1.55. With the complete database assembled, the numerical integration of equation 4 was performed with a $5^{\text {th }}$ order implicit Euler backward difference scheme. The line loads, mode shapes, modal frequencies and unsteady aerodynamic data at each time step are linearly interpolated between the data at the three Mach numbers.

The response is also calculated using linearized quasi-steady line loads. Linearization around the local mean $\alpha_{l}$, $\beta_{l}$ results in a state space model given by

$$
\{\dot{\chi}\}=[A]\{\chi\}+\left\{G_{g}\right\}+\left\{G_{e d}\right\} \quad, \quad\{\chi\}=\{g, \dot{g}\}^{T}
$$

where

$$
[A]=\left[\begin{array}{cc}
0 & I \\
-M^{-1} \Omega & -M^{-1} \Delta
\end{array}\right] .
$$

The definitions in equation 6 still apply, but the aerodynamic damping and stiffness matrices are given by equations 14 and 15 of reference [33], and the aerodynamic mass matrix is zero.

Because separate MSC.Nastran ${ }^{T M}$ eigenvalue analyses were performed on the structural models at each of the three flight conditions, the mode shape vectors were assigned differing signs by the software. Since it is possible to define

$$
[m]=[\phi][I][\phi]^{T} \quad, \quad[K]=[\phi]\left[\omega^{2}\right][\phi]^{T}
$$

the sign on eigenvectors is arbitrary. Accordingly, the signs of the mode shapes at Mach numbers 1.20 and 1.55 were changed so that mode 1 maintained the same sign orientation at all Mach numbers. This sign change too was necessary for a stable multi-Mach number simulation. Finally, what is expected to be a conservative approach to engine loading, engine gimballing is used at each time step to instantaneously trim the vehicle response to gust loading ${ }^{37}$.

\section{Gust Model}

In this study composite one-minus-cosine gust profiles typical of the Statistical Discrete Gust (SDG) method will be used. This method assumes that gusts can be built up as an assembly of discrete, smoothly varying ramp-hold functions ${ }^{38,39}$. Four one-minus-cosine ramps are used separated by hold functions. In the present analysis, gusts are modeled by adding a local angle of attack increment due to gust, given by

$$
\Delta \alpha_{g}=\frac{1}{2} \sum_{n=1}^{4} w_{r e f_{n}}\left(L_{g_{n}} / b_{g}\right)^{\kappa} \operatorname{Sgn}_{n}\left(1-\cos \left(\theta_{n}\right)\right) / U_{\infty}
$$

where

$$
\theta_{n}=\frac{\pi\left(t-t_{g_{n}}-x / U_{\infty}\right)}{L_{g_{n}} / U_{\infty}} \quad \text { for } \quad t_{g_{n}}+x / U_{\infty}<t<t_{g_{n}}+x / U_{\infty}+L_{g_{n}} / U_{\infty}
$$

and

$$
\theta_{n}=\pi \quad \text { for } \quad t \geq t_{g_{n}}+x / U_{\infty}+L_{g_{n}} / U_{\infty} .
$$

Figure 1 shows a typical velocity profile of a composite gust with 4 segments and the phase and width parameters $t_{g}$ and $L_{g}$ for segment 3 . The resulting local angle of attack increment due to gust produces a loading increment, computed from the steady rigid line loads database. Integration over the vehicle length produces the generalized force increment $G_{g}$ due to gust. This provides the forcing on the right hand side of equations 4 and 7 . The response to the gust is found in the evolution of the generalized variables and the lag states.

\section{Verification of Performance}

Figures 2-4 show the aerodynamic dampings and frequencies resulting from the present unsteady ROM and the FUN3D computational aeroelastic code separately computed at each of Mach numbers 1.00, 1.20 and 1.55. Note that aerodynamic damping as defined here is due to the motion of the aeroelastic modes and is a function of dynamic pressure. The unsteady ROM solutions are computed at $q_{\text {nom }}$ and $1.32 q_{\text {nom }}$ while time marching FUN3D solutions are 
shown at $q_{\text {nom }}$. Most of the solutions are at $\alpha=0.0$ degrees except the Mach 1.55 FUN3D time marching solution, which is at $\alpha=2.5$ degrees. (The aerodynamic damping at Mach 1.55, $\alpha=0.0$ and $\alpha=2.5$ degrees are similar.) In each case the frequency and aerodynamic damping values in these figures were obtained from a log decrement of the time response to an initial generalized modal velocity. Note that at Mach numbers 1.00 and 1.20 mode 1 is aerodynamically undamped and becomes further undamped at higher dynamic pressure. At Mach 1.55, mode 1 aerodynamics is damped and becomes further damped at higher dynamic pressure. With the exception of Mach 1.20 where the unsteady ROM model slightly over predicts mode 2 damping and mode 1 undamping, the unsteady ROM results compare very well with the time marching computational aeroelastic simulations. This initial verification indicates that individual ROM databases are consistent with the full order CFD solutions. The progression from negative aerodynamic damping in mode 1 to positive damping at higher Mach numbers is a feature that will be important in the simulations to follow.

Since a gust model is included in the reduced-order analyses, it is of interest to compare the gust responses of the reduced-order models with that from the FUN3D time marching gust simulation. Figures 5-6 show the generalized displacements and force time histories comparing Ares I CLV gust simulations using the full order FUN3D CFD and unsteady ROM methods. Figure 5 shows the Ares I generalized displacement and force time histories due to a sharp edge gust equivalent to a 2 degree angle of attack increment. In this case critical structural damping is used to damp out structural oscillations. Because of the time lag required for lift to be generated, there is a period of time over which the steady state displacement and forces develop. Although there are minor differences, the full order and reduced-order solutions compare quite well.

Figure 6 presents a somewhat more challenging case. The composite gust profile with four one-minus-cosine gust ramps, and associated time sequencing produces the response shown. The reduced and full order generalized displacement time histories match very well. The generalized force requires somewhat more discussion. The quasisteady and unsteady ROM's include the aerodynamic modal forcing implicitly in matrix $[A]$ of equations 5 and 8 . The gust forcing is included explicitly in the term $\left\{G_{g}\right\}$. Therefore, the unsteady ROM generalized force shown in each of Figures 5 and 6 are only the gust forcing ( $G_{g}$ in equations 4 and 7 ) while the full order FUN3D generalized force is due to all aerodynamic effects combined. Additional dynamics are therefore evident in the FUN3D time accurate solution that are not seen in the gust forcing increment in the ROM solution. But the displacements and forces give at least a qualitative agreement.

Having generally verified the performance of the model for individual flight conditions and the gust simulation methodology, the progression through flight conditions is now demonstrated. The mode 1 time history of the response to an initial generalized velocity excitation is presented in Figure 7. Here, a comparison is shown between the unsteady and the quasi-steady ROM's. These simulations traverse the entire Mach number range from 1.00 to 1.55 . Note that in the unsteady ROM the mode is initially undamped. Beyond Mach 1.20 mode 1 in the unsteady ROM simulation becomes aerodynamically damped. Comparing these results with the dampings of Figures 2-4, the aerodynamic damping of mode 1 at each Mach number is correctly reproduced in the unsteady ROM simulation. Also shown in Figure 7 is a quasi-steady simulation over the same Mach number range. The quasi-steady simulation results in a strongly damped mode 1 . This result is of course physically inaccurate, in that the quasi-steady approach does not include the dynamics resulting from the unsteady aerodynamics and associated time lags. The result is, though, consistent with the quasi-steady results shown previously ${ }^{34}$.

Finally the dynamic thrust gimballing used here is demonstrated in Figure 8. Note that as discussed earlier, the mean part of the solution is trimmed for changes in Mach number and dynamic pressure in a static sense. On the other hand, the linearized dynamic solution introduces the gust excitation into the solution. The gust excitation produces an instantaneous dynamic thrust angle change to trim at each time step. An instantaneous thrust angle change to trim is physically unrealistic since a control system will not fully trim the vehicle at each point in time. The present method however, can be considered conservative in that it will produce a higher bending moment than dynamic thrust control would produce ${ }^{37}$. Figure 8 shows the gust velocity excitation and the dynamic thrust gimbal angle resulting from that. The bending moment time history is also shown. The bending moment shown is measured at the streamwise midpoint of the vehicle. The introduction of thrust gimballing is clearly seen to increase the peak dynamic bending moment. 


\section{Example - Quantification of Uncertainty}

The quantities of interest are the uncertainty in the maximum bending moment, rms bending moment at the streamwise midpoint of the Ares I launch vehicle, and rms acceleration at the Crew Exploration Vehicle (CEV). To compute uncertainties in bending moment due to variations in flight and design parameters, a total of 32,000 computations have been performed using the quasi-steady and 32,000 using the unsteady ROM's. These solutions comprise all possible combinations of the chosen flight, design and gust parameters. Each simulation was exposed to a gust profile composed of four one-minus-cosine shapes spaced out by four time start parameter values. Three sets of gust shape signs were used. The gust time starts $\left(t_{g_{n}}, n=1,4\right)$ were varied between three sets of four values. For maximum launch vehicle loading due to gust, a $350 \mathrm{in} / \mathrm{sec}$ one-minus-cosine wave form was tuned to the lower frequency modes by setting the maximum value of $L_{g_{n}}$. Standard atmospheric data suggests this will give a 99 percent closure and 90 percent confidence level loading ${ }^{40}$. The gust magnitudes $\left(w_{\text {ref }}, n=1,4\right)$ take on three sets of four values, between 100 and $350 \mathrm{in} / \mathrm{sec}$. The gust width parameters $\left(L_{g_{n}}, n=1,4\right)$ took eight sets of four values variously spaced between minimum and maximum one-minus-cosine lengths.

Computations with all of these parametric variations were performed both for the purely quasi-steady and the unsteady ROM's. Furthermore, to assess the likelihood of a given maximum bending moment value, Gaussian probability distributions centered about a nominal value have been assigned to each of the parameters. Gust velocities are also assigned probabilities reflecting the atmospheric data of reference [40]. (For instance, a $350 \mathrm{in} / \mathrm{sec}$ gust is given here a probability of approximately 1 percent.) In this paper the total probability of an event is the product of probabilities of each of the design, flight and gust parameters.

Illustrative of the results obtained by these simulations, Figure 9 shows the total bending moment time history for the most extreme condition, computed with both the quasi-steady and the unsteady ROM's. This condition is the case giving the largest maximum total bending moment. Total bending moment is the combination of the static mean and dynamic bending moment components. The dynamic component is caused by the gust excitation plus required thrust gimballing to trim during gust. The parameters resulting in this case are the highest dynamic pressure, lowest first mode frequency, lowest structural damping, highest gust velocities with best tuned width. Note that for this case, the dynamic component dwarfs the static mean component of bending moment. The gust excitation is completed within the first 2 seconds of the simulation while the flexible response continues much longer. The largest absolute magnitude of bending moment in the quasi-steady simulation occurs at about 2.5 seconds while the largest absolute magnitude of bending moment in the unsteady simulation occurs at about 9 seconds into the simulation. Because of the unsteady aerodynamics of mode 1, the unsteady ROM results, over the entire course of the simulation, in larger excursions of bending moment than the quasi-steady simulation. The response of mode 1 with the quasi-steady ROM remains damped through out. Even though the unsteady ROM gives a 15 percent larger maximum bending moment than does the quasi-steady ROM, the probability of the combination of parameters producing this result is extremely small, well beyond a 99 percent closure requirement.

Figure 10 presents outlines of the histograms of the maximum total bending moment for every combination of design, flight and gust parameters. The resulting values are assigned among 150 bins. Typical histograms show bar plots, however, in the present case, bars would obscure the comparison of the two sets of results. Therefore, only the outlines of the histograms are shown. Summed probabilities for all occurances of each maximum bending moment value form the outlines of the histograms shown. The quasi-steady and unsteady ROM's have nearly the same distribution of maximum bending moment. As just discussed, only in the most extreme and most improbable cases is there a significant difference in maximum bending values. The 95 percent values of maximum bending moment are nearly identical for the two methods. The 95 percent value of the rms bending moment, as indicated in the histogram outlines of Figure 11 computed with the unsteady ROM is significantly higher than that computed with the quasisteady method. This is indicative of the greater persistance of the mode 1 oscillation for the unsteady ROM. Figure 12 shows the histogram outline of the rms $z$-acceleration at the CEV. The persistance of mode 1 dynamics is also evident in the higher 95 percent acceleration level experienced at the crew module.

\section{Concluding Remarks}

This paper has presented an approach to simulating flexible launch vehicle ascent through multiple flight conditions. The approach includes a simultaneous computation of a nonlinear mean solution as well as a linearized dynamic solution. Although currently restricted only to flexible vehicle modes, the approach is applicable to rigid body degrees of freedom as well. The approach shown here is verified by comparing aerodynamic damping at three transonic Mach 
numbers. A gust model is also included in the reduced-order model, and verified by comparison with a direct CFD simulation of a gust. Dynamic and quasi-steady reduced-order model databases are constructed for three Mach numbers through the transonic regime. The dynamics of the flexible vehicle is compared for the two methods. An example of the use of these reduced-order models and multi-flight condition databases, the uncertainty in the maximum bending moment, rms bending moment at the streamwise midpoint of the Ares I launch vehicle, and rms acceleration at the crew module are computed for ascent traversing the transonic regime. The resulting probability distributions show that the unsteady reduced-order model results in higher 95 percent rms vehicle bending moment and crew module acceleration than the quasi-steady reduced-order model.

\section{References}

${ }^{1}$ Capri, F., Mastroddi, F., and Pizzicaroli, A., "Linearized Aeroelastic Analysis for a Launch Vehicle in Transonic Flight," Journal of Spacecraft and Rockets, Vol. 43, No. 1, 2006, pp. 92-104.

${ }^{2}$ Silva, W., Vatsa, V., and Biedron, R., "Reduced order models for the aeroelastic analysis of the Ares Vehicles," AIAA Paper 2010-4375, June 2010.

${ }^{3}$ Mastroddi, F., Stella, F., Cantiani, D., and Vetrano, F., "Linearized Aeroelastic Gust Response of a Launch Vehicle," Journal of Spacecraft and Rockets, Vol. 48, No. 3, 2011, pp. 420-432.

${ }^{4}$ Chen, P. C., Baldelli, D. H., and Zeng, J., "Dynamic Flight Simulation (DFS) Tool for Nonlinear Flight Dynamic Simulation Including Aeroelastic Effects," AIAA Atmospheric Flight Mechanics Conference, No. AIAA-2008-6376, 2008.

${ }^{5}$ Amsallem, D., Cortial, J., and Farhat, C., "Toward Real-Time Computational-Fluid-Dynamcs-Based Aeroelastic Computations Using a Database of Reduced-Order Information,” AIAA Journal, Vol. 48, No. 9, 2010, pp. 2029-2037.

${ }^{6}$ Amsallem, D., Cortial, J., and Farhat, C., "On-Demand CFD-Based Aeroelastic Predictions Using a Database of Reduced-Order Bases and Models," 47th AIAA Aerospace Sciences Meeting and Exhibit, No. AIAA 2009-800, 2009.

${ }^{7}$ Amsallem, D., Cortial, J., Carlberg, K., and Farhat, C., "On-Demand CFD-Based Aeroelastic Predictions Using a Database of ReducedOrder Bases and Models," 50th AIAA/ASME/ASCE/ASC Structures, Structural Dynamics, and Materials Conference, No. AIAA 2009-2432, 2009.

${ }^{8}$ Singh, G., Ocampo, J., Acosta, C. A., and Millwater, H., "Distributed Computing for Probabilistic Structural Integrity Analysis of Aircraft Structures," 51 st AIAA/ASME/ASCE/ASC Structures, Structural Dynamics and Materials Conference, No. AIAA-2010-2848, 2010.

${ }^{9}$ Paez, T. L. and Mayes, R. L., "Uncertainty Quantification in Verification and Validation of Computational Solid Mechanics Models Example," 50th AIAA/ASME/ASCE/ASC Structures, Structural Dynamics and Materials Conference, No. AIAA-2009-2246, 2009.

${ }^{10}$ Ghanem, R., R.-h. J., Benjamin, A., Doostan, A., and Zou, Y., "Stochastic Process Model for Material Properties Under Incomplete Information," 48th AIAA/ASME/ASCE/ASC Structures, Structural Dynamics and Materials Conference, No. AIAA-2007-1948, 2007.

${ }^{11}$ Urbina, A., Paez, T. L., Hasselman, T. K., Wathugala, G. W., and Yap, K., "Assessment of Model Accuracy Relative to Stochastic System Behavior," 44th AIAA/ASME/ASCE/ASC Structures, Structural Dynamics and Materials Conference, No. AIAA-2003-1617, 2003.

${ }^{12}$ Sarkar, A. and Ghanem, R., "Mid-Frequency Structural Dynamics With Parameter Uncertainty," 42nd AIAA/ASME/ASCE/ASC Structures, Structural Dynamics and Materials Conference, No. AIAA-2001-1454, 2001.

${ }^{13}$ Eldred, M. S. and Webster, C. G., "Design Under Uncertainty Employing Stochastic Expansion Methods," 12th AIAA/ISSMO Multidisciplinary Analysis and Optimization Conference, No. AIAA-2008-6001, 2008.

${ }^{14}$ Swiler, L. P., Adams, B. M., and Eldred, M. S., "Model Calibration Under Uncertainty: Matching Distribution Information," 12th AIAA/ISSMO Multidisciplinary Analysis and Optimization Conference, No. AIAA-2008-5944, 2008.

${ }^{15}$ Lockwood, B. A., Rumpfkeil, M. P., Yamazaki, W., and Mavriplis, D. J., "Uncertainty Quantification in Viscous Hypersonic Flows Using Gradient Information and Surrogate Modeling," 49th AIAA Aerospace Sciences Meeting and Exhibit, No. AIAA 2011-885, 2011.

${ }^{16}$ Wang, Q., Ham, F., Iaccarino, G., and Moin, P., "Risk Quantification in Unsteady Flow Simulations using Adjoint-based Approaches," 50th AIAA/ASME/ASCE/ASC Structures, Structural Dynamics and Materials Conference, No. AIAA-2009-2277, 2009.

${ }^{17}$ Rumpfkeil, M. P., Yamazaki, W., and Mavriplis, D. J., "A Dynamic Sampling Method for Kriging and Cokriging Surrogate Models," 49th AIAA Aerospace Sciences Meeting and Exhibit, No. AIAA 2011-883, 2011.

${ }^{18}$ Bose, D., Wright, M. J., and Palmer, G. E., "Uncertainty Analysis of Laminar Aeroheating Predictions for Mars Entries," Journal of Thermophysics and Heat Transfer, Vol. 20, No. 4, 2006, pp. 652-662.

${ }^{19}$ Danowsky, B. P., Chrstos, J. R., Klyde, D. H., Farhat, C., and Brenner, M., "Evaluation of Aeroelastic Uncertainty Analysis Methods," Journal of Aircraft, Vol. 47, No. 4, 2010, pp. 1266-1273.

${ }^{20}$ Bartels, R. E., "A Quasi-steady Flexible Launch Vehicle Stability Analysis Using Steady CFD with Unsteady Aerodynamic Enhancement," Journal of Spacecraft and Rockets, 2012, accepted for publication.

${ }^{21}$ Anderson, W. K., Rausch, R. D., and Bonhaus, D. L., "An Implicit Upwind Algorithm for Computing Turbulent Flows on Unstructured Grids," Computers and Fluids, Vol. 23, No. 1, 1994, pp. 1-22.

${ }^{22}$ NASA LaRC, Hampton, VA, FUN3D Manual, November 2008, http: / f fun3d. larc. nasa.gov.

${ }^{23}$ Spalart, P. R. and Allmaras, S. R., "One-Equation Turbulence Model for Aerodynamic Flows," 30th AIAA Aerospace Sciences Meeting and Exhibit, No. 439, 1992.

${ }^{24}$ Vatsa, V. N. and White, J. A., "Calibration of a Unified Flux Limiter for Ares-Class Launch Vehicles from Subsonic to Supersonic Speeds," 56th Propulsion Meeting 5, JANNAF, III, April 2009.

${ }^{25}$ Nielsen, E. J., Lu, J., Park, M. A., and Darmofal, D. L., "An Exact Dual Adjoint Solution Method for Turbulent Flows on Unstructured Grids," Computers and Fluids, Vol. 33, No. 9, 2004, pp. 1131-1155.

${ }^{26}$ Raveh, D. E., "CFD-Based Models of Aerodynamic Gust Response," 47th AIAA/ASME/ASCE/AHS/ASC Structures, Structural Dynamics, and Materials Conference, No. 2022, 2006. 
${ }^{27}$ Zaide, A. and Raveh, D. E., "Numerical Simulation and Reduced-Order Models for Gust-Response Analysis of Flexible Wings," 17th AIAA Fluid Dynamics Conference, 2005.

${ }^{28}$ Parameswaran, V. and Baeder, J. D., "Indicial Aerodynamics in Compressible Fluid Dynamic Calculations," Journal of Aircraft, Vol. 34, No. 1, 1997, pp. 131-133.

${ }^{29}$ Raveh, D. E., "Gust Response Analysis of Free Elastic Aircraft in the Transonic Flight Regime," Journal of Aircraft, Vol. 48, No. 4, 2011, pp. 1204-1211.

${ }^{30}$ Zaide, A. and Raveh, D. E., “Numerical Simulation and Reduced-Order Modeling of Airfoil Gust Response,” AIAA Journal, Vol. 44, No. 8, 2006, pp. 1826-1834.

${ }^{31}$ Raveh, D. E., "CFD-Based Gust Response Analysis of Free Elastic Aircraft," ASD Journal, Vol. 2, No. 1, 2010, pp. $23-34$.

${ }^{32}$ Wang, Z., Zhang, Z., Chen, P. C., and Sarhaddi, D., "A Compact CFD-Based Reduced Order Modeling for Gust Analysis," 52th AIAA/ASME/ASCE/ASC Structures, Structural Dynamics and Materials Conference, No. AIAA-2011-2041, 2011.

${ }^{33}$ Bartels, R. E., Chwalowski, P., Massey, S. J., Heeg, J., and Mineck, R. E., "Computational Aeroelastic Analysis of the Ares Crew Launch Vehicle During Ascent," 28th AIAA Applied Aerodynamics Conference, No. AIAA-2010-4374, 2010.

${ }^{34}$ Bartels, R. E., Chwalowski, P., Massey, S. J., Heeg, J., and Mineck, R. E., "Computational Aeroelastic Analysis of the Ares I Crew Launch Vehicle During Ascent," Journal of Spacecraft and Rockets, 2012, accepted for publication.

${ }^{35}$ Roger, K. L., "Airplane Math Modeling Methods for Active Control Design," AGARD Structures and Materials Panel, No. CP-228, 1977, pp. 4-1..4-11.

${ }^{36}$ Bartels, R. E., "A Quasi-Steady Flexible Launch Vehicle Stability Analysis Using Steady CFD with Unsteady Aerodynamic Enhancement," 49th AIAA Aerospace Sciences Meeting, No. AIAA-2011-1114, 2011.

${ }^{37}$ Kabe, A. M., "Design and Verification of Launch and Space Vehicle Structures," No. AIAA-98-1718, 1998.

${ }^{38}$ Jones, J. G., "An Equavalence Between Deterministic and Probablistic Design Criteria For Linear Systems," Journal of Sound and Vibration, Vol. 125, No. 2, 1988, pp. 341-356.

${ }^{39}$ Perry III, B., Pototzky, A. S., and Woods, J. A., "NASA Investigation of a Claimed "Overlap" Between Two Gust Response Analysis Methods," Journal of Aircraft, Vol. 27, No. 7, 1990, pp. 605-611.

${ }^{40}$ Adelfang, S. I. and Smith, O. E., "Gust Models for Launch Vehicle Ascent," 36th AIAA Aerospace Sciences Meeting and Exhibit, No. AIAA-98-747, 1998.

${ }^{41}$ Cordova, H. S., Leahy, F. B., Adelfang, S. I., Roberts, B. C., Barbre, R. E., Starr, B. R., Puperi, D. S., and Duffin, P. F., "Use of Smoothed Measured Winds to Predict and Assess Launch Environments," AIAA Atmospheric Flight Mechanics Conference, No. AIAA-2011-6464, 2011. 


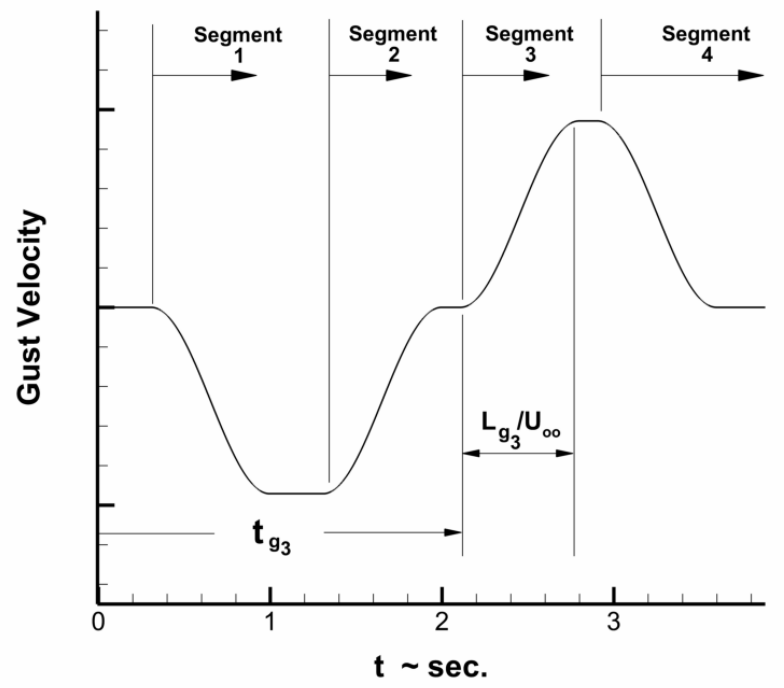

Figure 1. Gust velocity profile, ( $\operatorname{Sgn}$ values are $-1,1,1,-1)$.

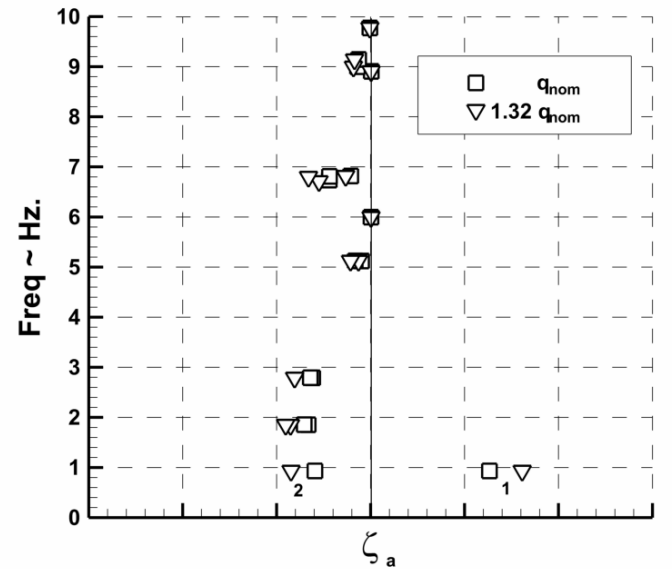

(a) Unsteady ROM.

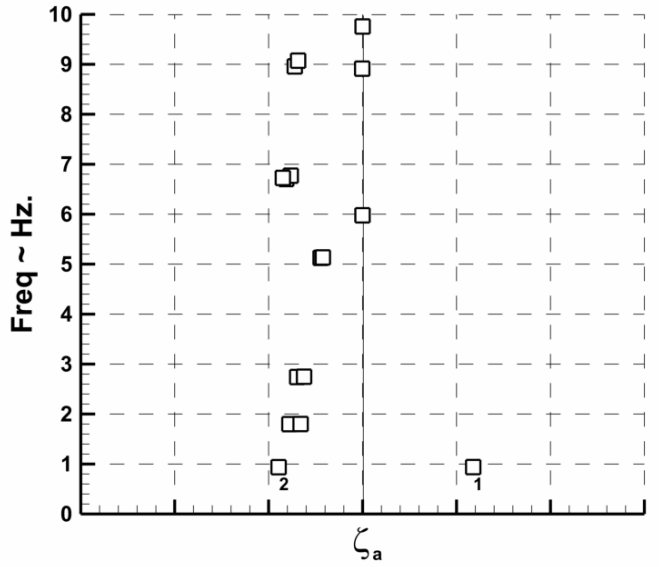

(b) FUN3D time marching, $q_{\text {nom }}$.

Figure 2. Aerodynamic damping versus frequency, Mach 1.00, $\alpha=0.0$ deg.. 


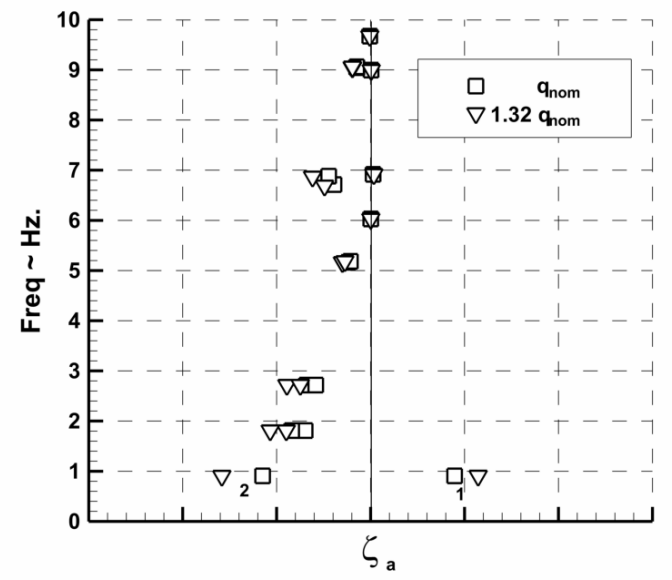

(a) Unsteady ROM.

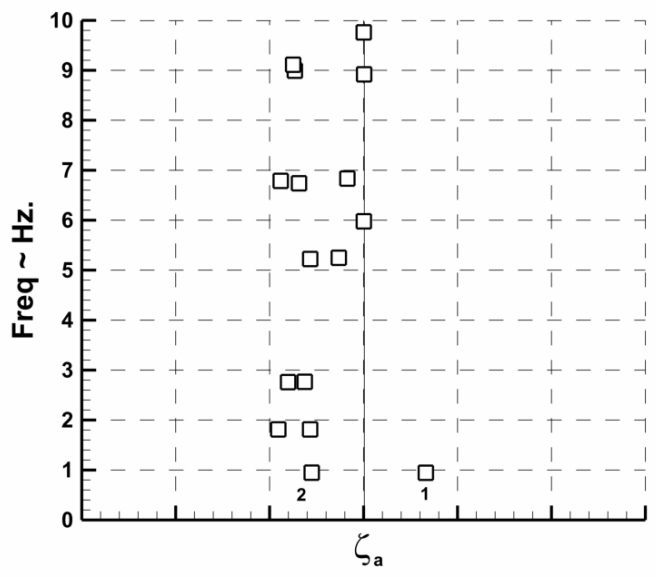

(b) FUN3D time marching, $q_{\text {nom }}$.

Figure 3. Aerodynamic damping versus frequency, Mach $1.20, \alpha=0.0$ deg..

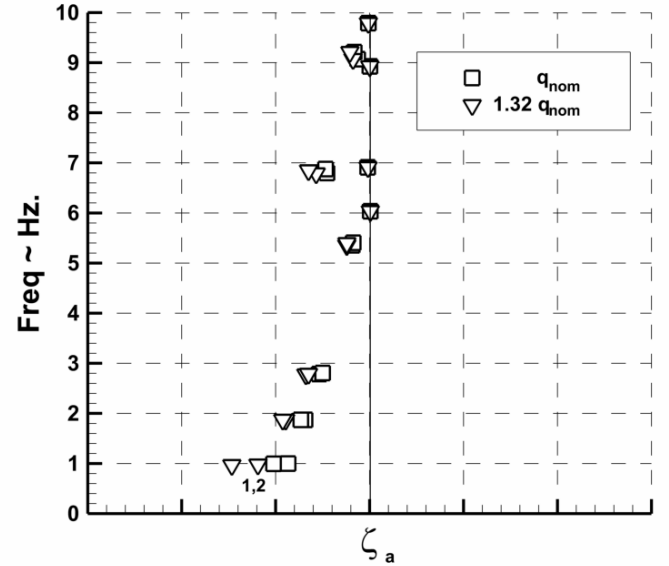

(a) Unsteady ROM, $\alpha=0.0$ deg..

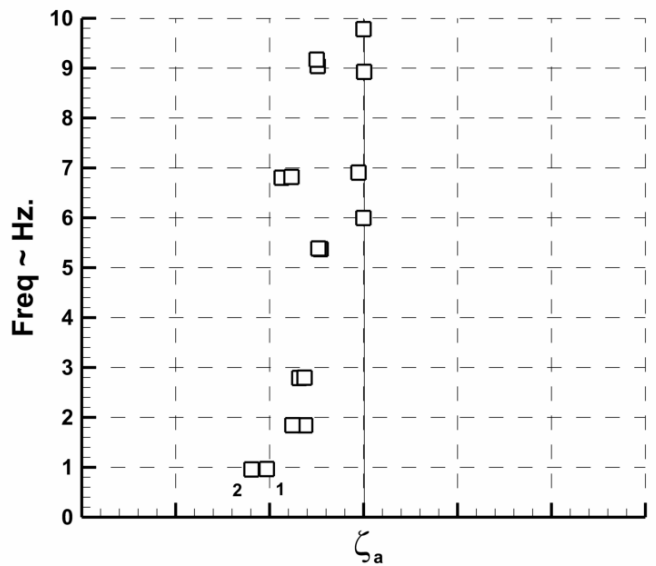

(b) FUN3D time marching, $\alpha=2.5$ deg., $q_{\text {nom }}$.

Figure 4. Aerodynamic damping versus frequency, Mach 1.55. 

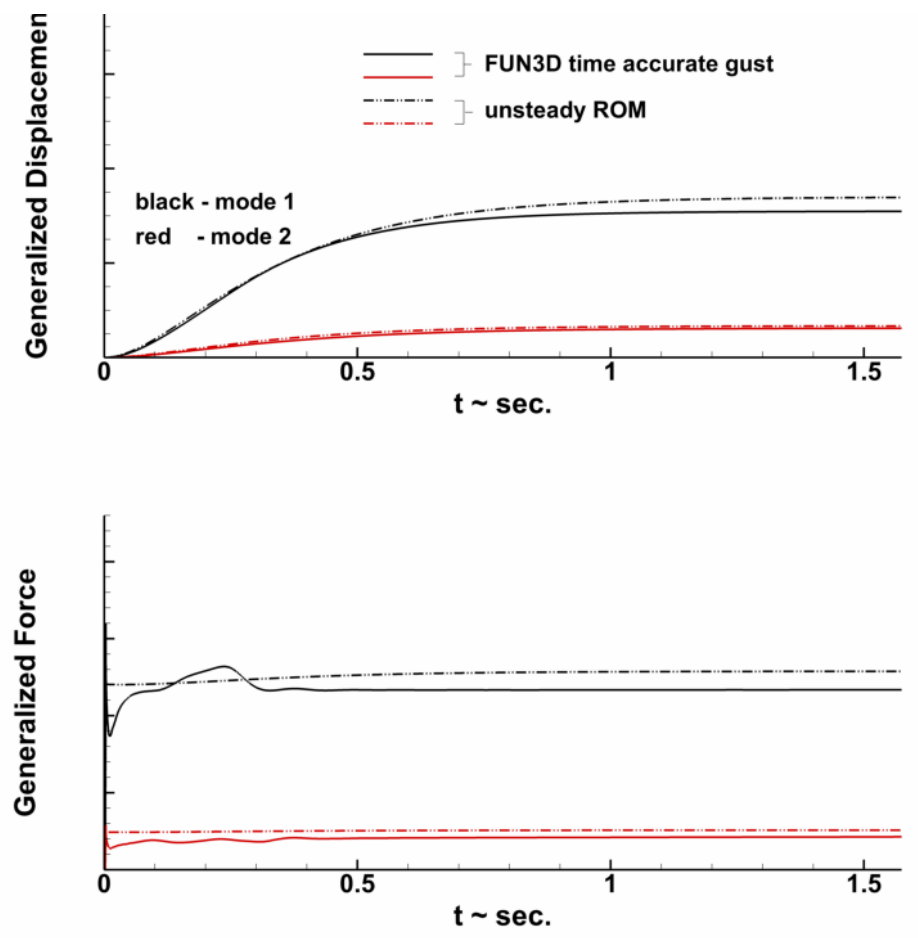

Figure 5. Generalized displacement and force time histories due to a sharp edged 2 degree gust, Mach 1.00, $\alpha=0.0$ deg..
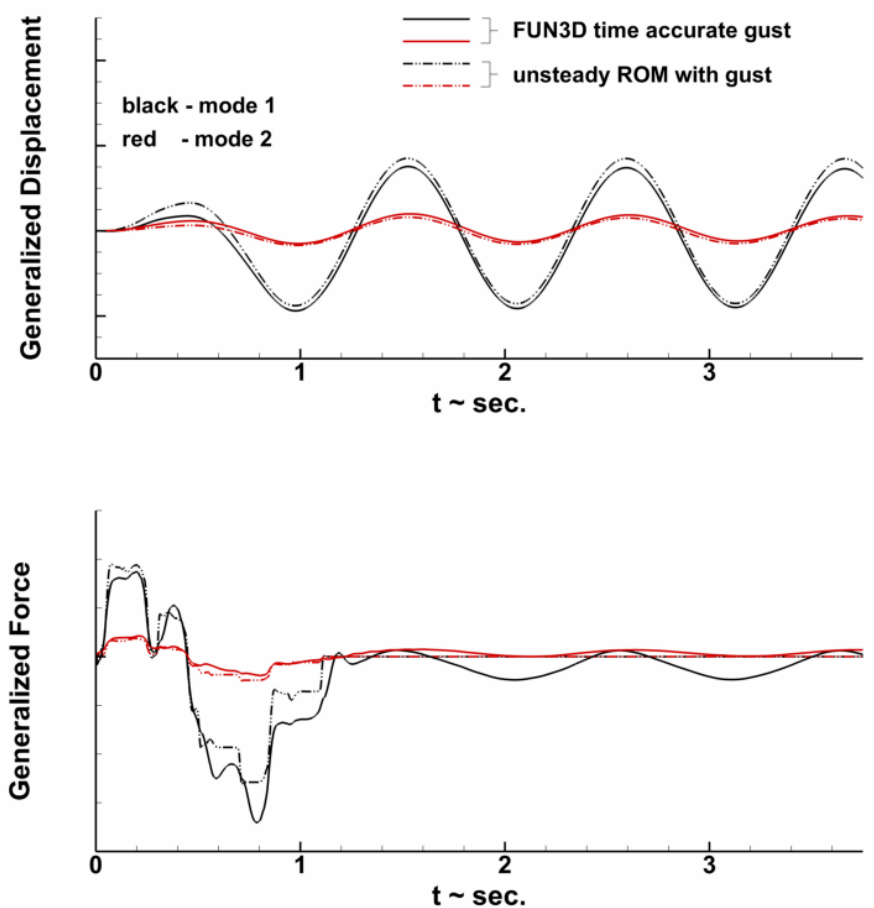

Figure 6. Generalized displacement and force time histories due to composite one-minus-cosine gust, Mach 1.00, $\alpha=0.0$ deg..

12 of 16 


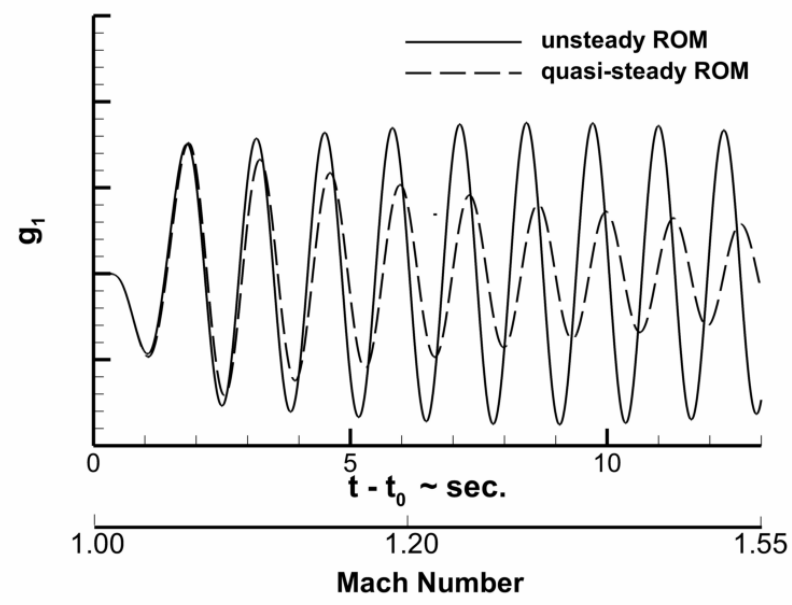

Figure 7. Mode 1 generalized displacement time history due to initial generalized velocity. 


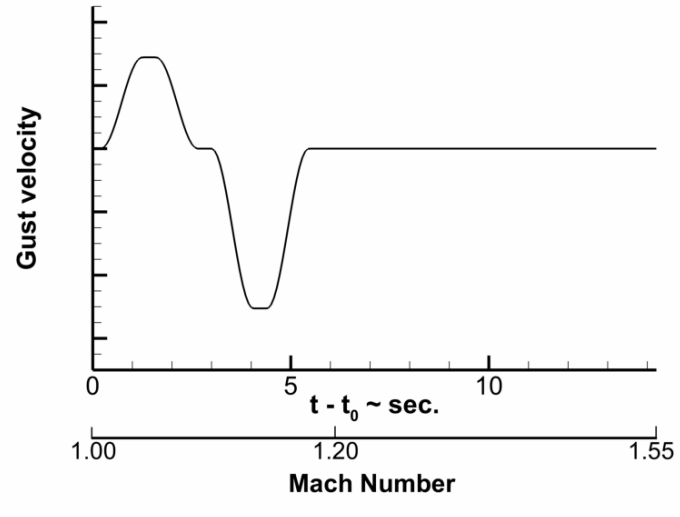

(a) Gust velocity.

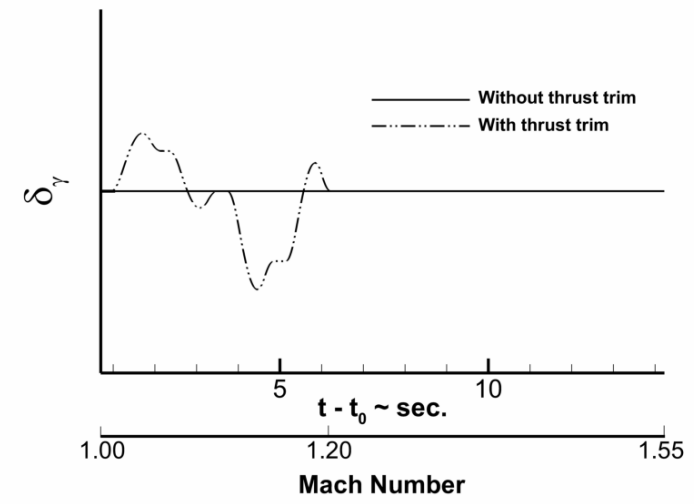

(b) Dynamic component of engine gimbal angle.

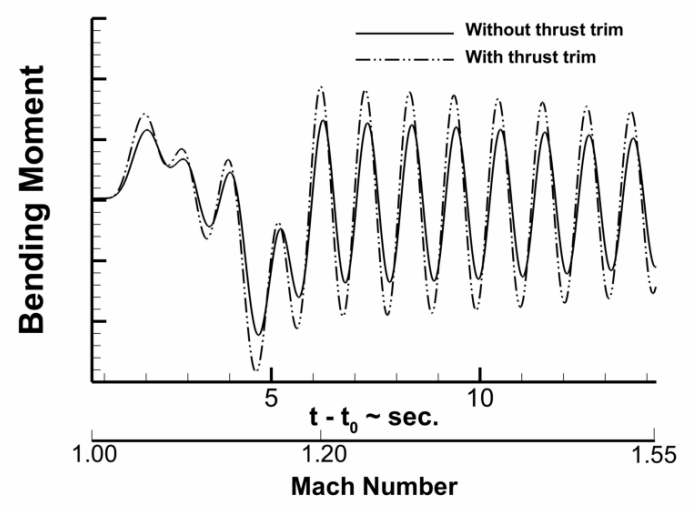

(c) Dynamic component of bending moment.

Figure 8. Effect of thrust gimbaling to trim. 


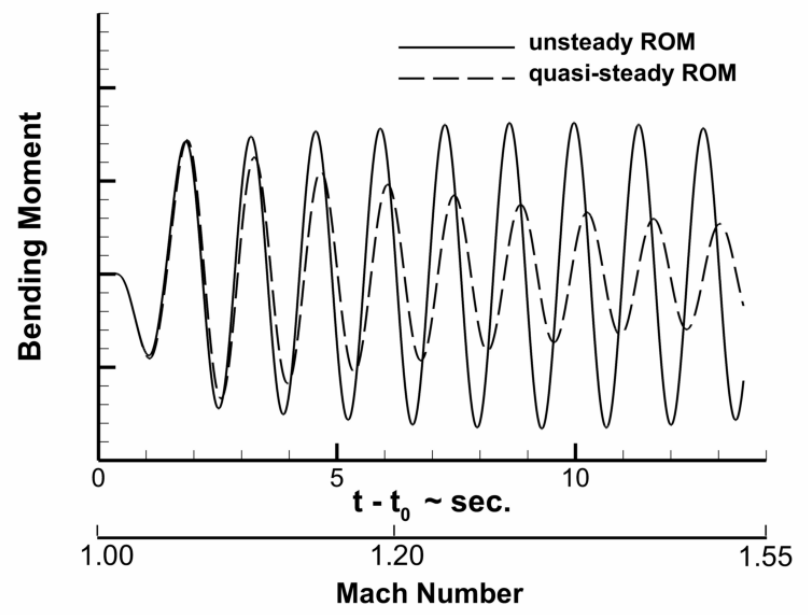

Figure 9. Time history of total bending moment. Most extreme loading case.

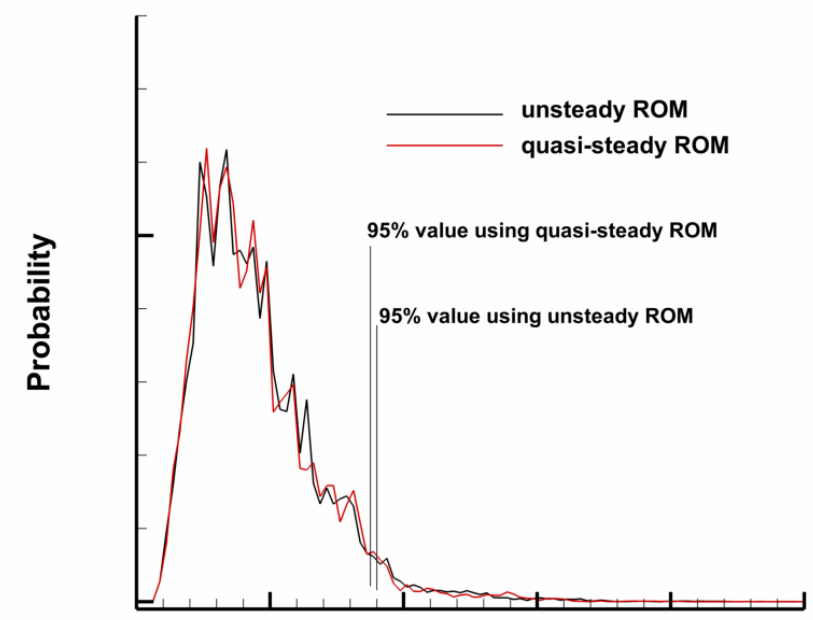

Max Bending Moment

Figure 10. Probability distribution of maximum total bending moment. 


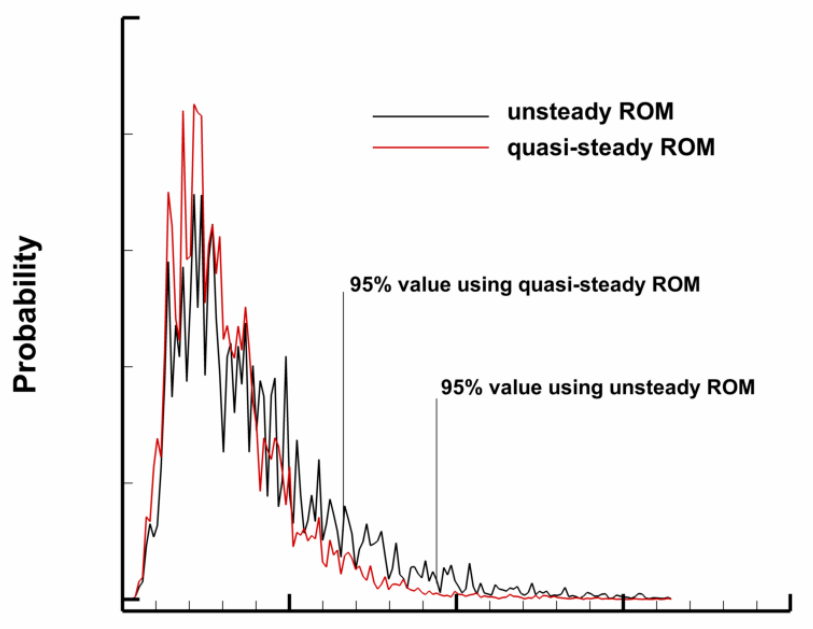

\section{RMS Bending Moment}

Figure 11. Probability distribution of rms total bending moment.

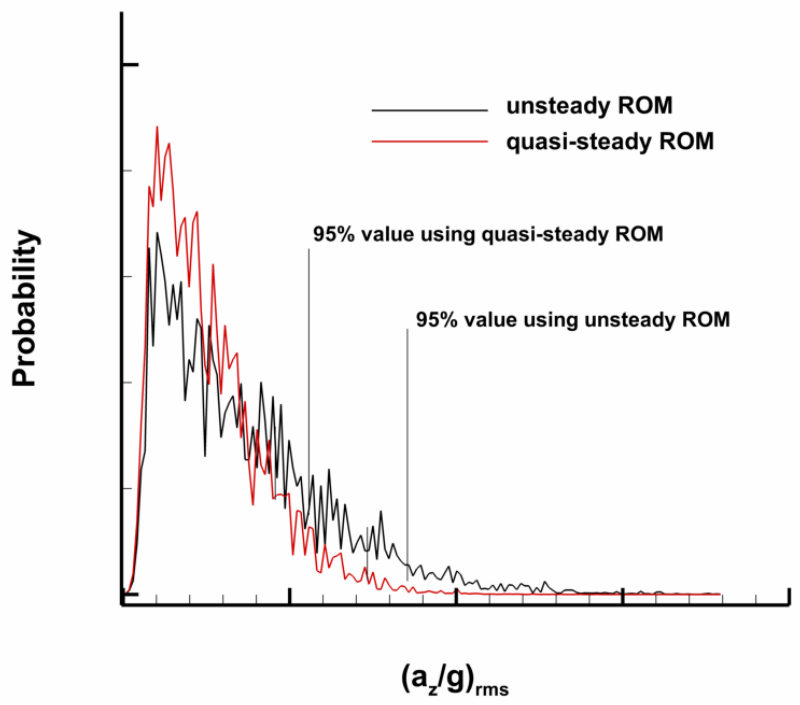

Figure 12. Probability distribution of $\mathbf{r m s} z$-dir. acceleration at CEV. 